\title{
THE IMPACT OF OPEN INGUINAL HERNIA MESH REPAIR ON QUALITY OF LIFE
}

\author{
Elif Cengiz ${ }^{1}$, Toprak Kağan Aksu ${ }^{1}$, Hilal Sena Çifcibaşs ${ }^{1}$, Tuğrul Demirel ${ }^{2}$
}

\author{
${ }^{1}$ Trakya University School of Medicine, Edirne, TURKEY \\ ${ }^{2}$ Department of General Surgery, Trakya University School of Medicine, Edirne, TURKEY
}

\begin{abstract}
Aims: This study aims to evaluate the effect of open inguinal hernia repair with mesh on the quality of life of the patients who were operated at a university hospital. Methods: In this cohort study, 86 patients who had undergone an open inguinal hernia repair at General Surgery Department in a university hospital between January 2017 and October 2019 were asked to fill out the Carolinas Comfort Scale questionnaire and the data were analyzed retrospectively. Results: The total number of patients in the study was 86 . Seventy-three were male (84\%) and 13 were female (16\%). The median age was 53 years ranging from 18 to 82 . The difference of pre- and post-operative scores revealed high significance in all categories and in total; laying down, bending over, sitting up, performing activities of daily life, coughing or deep breathing, walking or standing, walking up or down the stairs, exercising and total score. Conclusion: Inguinal hernia decreases the quality of daily life by limiting the movements with groin pain. Surgical low-tension repair with mesh improves the quality of life significantly. Keywords: Inguinal hernia, quality of life, mesh repair
\end{abstract}

\section{INTRODUCTION}

Inguinal hernia $(\mathrm{IH})$ is the most common of abdominal hernias with a $75 \%$ rate according to the National Health Service of the United Kingdom (1). The prevalence of $\mathrm{IH}$ is $1.7 \%$ in all ages and $4 \%$ for people who are aged over 45 (1). About $90 \%$ of IH patients are male whereas only $10 \%$ are female (2). The main risk factors of IH are family history, age, gender, collagen diseases, and high Body Mass Index (3). Even though there's evidence to suggest that genetics may play a role in having congenital $\mathrm{IH}$, most of the $\mathrm{IH}$ cases are acquired (2). Two-thirds of IH patients are symptomatic and the main complaints are protrusion and pain in the groin (3). Generally, the symptoms and a precise physical examination are sufficient for the diagnosis (2).

Several repair techniques for $\mathrm{IH}$ are open repair with suture (Shouldice), open repair with mesh (Lichtenstein) and laparoscopic extraperitoneal repair with mesh (TEP) or transabdominal preperitoneal repair with mesh (TAPP) (4). Surgical repair is the most efficient treatment in $\mathrm{IH}$ and mesh repair is the gold standard treatment $(2,3)$. In non-mesh tension repair, sutures put tension on either side of the defect in order to keep it closed thus the tension inhibits the complete healing of the edges (5). Whereas in mesh repair, the mesh acts as a bridge between the sides of the defect thus decreases the tension. With the tension-free nature of the mesh repair, the recurrence rate and the rehabilitation period is reduced compared to non-mesh tension repairs (6).

Quality of life (QoL) after hernia repair is a common point of interest. Both the technical differences and also different meshes are evaluated concerning their impact especially on movement ability and groin pain in different studies. Rutegard et al. (7) found no statistical difference between light or heavyweight polypropylene mesh patients regarding QoL scores. Sanders et al. (8) reported no significant superiority of mesh fixation technologies including fibrin sealants, self-fixing meshes and NB2C glues over conventional suture fixation on postoperative quality of life. 
In the routine practice, $2 \mathrm{D}$ polypropylene single layer standard meshes are being used for open inguinal hernia repairs with suture fixation in our state university hospital setting for social security insurance patients.

The aim of this study is to compare the preoperative and postoperative quality of life of the patients who have undergone an open inguinal hernia repair with polypropylene mesh at the General Surgery Department between January 2017 and October 2019.

\section{MATERIAL AND METHODS}

This retrospective cohort study was approved by the Scientific Research Ethics Committee of Trakya University Medical Faculty (Protocol Code: TÜTF-BAEK2019/384). 190 patients were included in the study who have undergone an open inguinal hernia surgery at General Surgery Department between January 2017 and October 2019. Only 86 patients who were reached included in this study. Patients were asked to fill out the Carolinas Comfort Scale (CCS) questionnaire by phone call for preoperative and postoperative conditions. The questionnaire consists of 23 questions that measure pain, movement limitations and sensation of the mesh in 8 different categories listed in Table 1. In the preoperative period, the sensation of the hernia sac is questioned instead of the sensation of mesh. Each question carries points on a scale from 0 to 5 . At 0 points, no symptoms were seen, while 5 points represented the worst symptoms. The best score (patient with no symptoms) is 0 and the worst score (patient with the worst symptoms) was 115 (9).

The data were analyzed with IBM SPSS version 23.0.0.0. All categories and total scores were analyzed using the Shapiro-Wilk test for normal distribution. Non-normal distribution was observed for each 8 different categories and total score. The Wilcoxon test was used to compare the scores given to each question before and after surgery for all categories and total scores. A p-value of $<0.05$ was evaluated as statistically significant. Numbers, percentages, median, minimum, maximum variables, 1 st quartile and 3 rd quartile were used as descriptive statistics for this study.

\section{RESULTS}

In this retrospective cohort study, 86 patients who had undergone an open inguinal hernia repair were included after 1-month postoperatively. Not all the patients completed the whole questionnaire, with a sum of 11 patients having some questions missing. However, we conducted the study on a parameter basis of total of 8 parameters. Patients who had given missing data were not included in total score, but they were included in the parameters that they participated in questionnaires (Table 2). Seventy-three (84.9\%) patients were male and thirteen $(15.1 \%)$ were female. All patients' median age was 53 years. The minimum patient age was 18 years and the maximum was 82 years. There was a statistically significant difference between preoperative and postoperative scores of each category (laying down, bending over, sitting up, performing activities of daily living, coughing or deep breathing, walking or standing, walking up or down the stairs, exercising) and the total score $(\mathrm{p}<0.001)$ (Table 2). The descriptive statistics (median, minimum, maximum variables, 1st quartile and 3rd quartile) are presented in Table 2.

\section{DISCUSSION}

In this study, the change in quality of life of the patients who had undergone an open inguinal hernia repair with polypropylene mesh at the General Surgery Department between January 2017 and October 2019, is analyzed. Inguinal hernias are one of the most common afflictions of adults, especially for men (10). Its age and sex distributions are one of the most important epidemiological bases (11). Burcharth et al. (12) found in their study, $88.6 \%$ of the patients who had undergone an IH operation are male and $11.4 \%$ are female. In our study, there were 73 (84.9\%) male and 13 (15.1\%) female patients. Similarly, Primatesta et al. (11) reported 27924 inguinal hernia repairs with $91 \%$ male dominance. In both studies, it has been shown that inguinal hernia repair prevalence is higher for males than females in all age groups. Moreover, the lifetime risk of developing an inguinal hernia was found $27 \%$ for men and $3 \%$ for women by Öberg et al. (13). Therefore, a gender-dependent change in the prevalence of inguinal hernia can be stated. The natural pathway of testes from intraabdominal origin to scrotal location, forms a natural anatomical weakness of inguinal region in males. This might be a triggering factor for men having a higher incidence of inguinal hernia. Regarding the relation between age and inguinal hernia, it has been found that the inguinal re- 
Table1: Carolinas Comfort Scale questionnaire (7).

Question Score

\section{While laying down, do you have}

Sensation of mesh $\quad 012345$

$\begin{array}{ll}\text { Pain } & 012345\end{array}$

While bending over, do you have

$\begin{array}{ll}\text { Sensation of mesh } & 012345\end{array}$

Pain 012345

Movement limitations $\quad 012345$

While sitting up, do you have

Sensation of mesh $\quad 012345$

Pain 012345

Movement limitations $\quad 012345$

While performing activities of daily living (getting

out of bed, bathing, getting dressed), do you have

Sensation of mesh $\quad 012345$

Pain 012345

Movement limitations $\quad 012345$

When coughing or deep breathing, do you have

$\begin{array}{ll}\text { Sensation of mesh } & 012345\end{array}$

Pain $\quad 012345$

Movement limitations $\quad 012345$

When walking or standing, do you have

Sensation of mesh $\quad 012345$

Pain $\quad 012345$

Movement limitations $\quad 012345$

When walking up or down the stairs, do you have

$\begin{array}{ll}\text { Sensation of mesh } & 012345\end{array}$

Pain 012345

Movement limitations $\quad 012345$

When exercising (other than work-related), do you have

Sensation of mesh $\quad 012345$

Pain 012345

Movement limitations $\quad 012345$ 
Table 2: Summary of descriptive statistics and $p$ values of 8 different categories and total score.

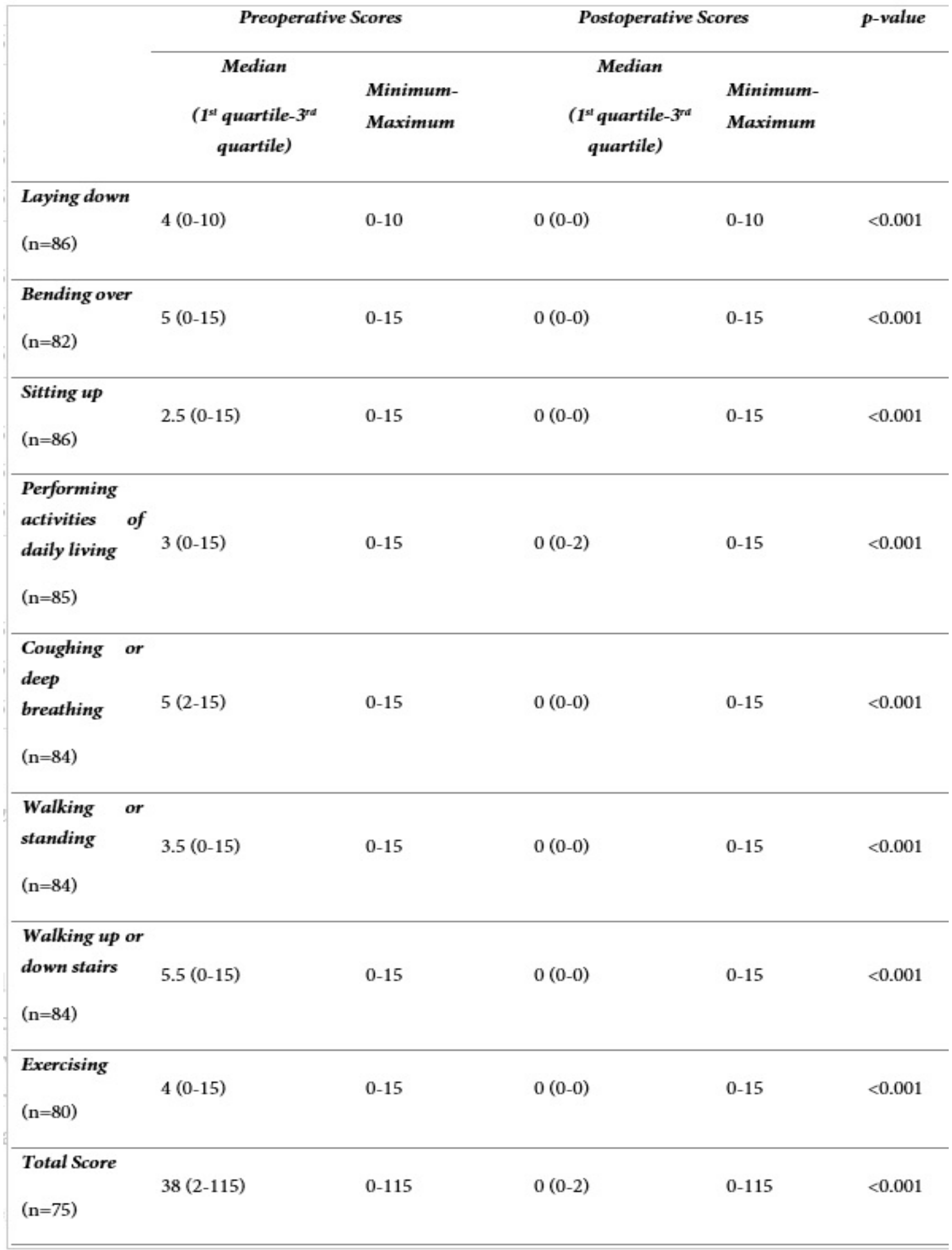


pair peaks bimodally at early childhood and old age for both sexes (12). Primatesta et al. (9) showed that from 2738 emergency admissions with an operation on inguinal hernia, $573(21 \%)$ were performed on infants and 1133 (41\%) on patients $\geq 65$ years. They also found that rates for elective surgery had increased up to late middle-age after a peak in infants and decreased slightly at elderly ages (11). However, there are no patients under 18 in our study.

In the current trial, 46 questions asked in total consisting of 23 questions about preoperative and 23 questions about postoperative periods. The questions included 8 main categories such as laying down, bending over, sitting up, performing activities of daily living, coughing or deep breathing, walking or standing, walking up or down the stairs, exercising. A high score meant the worse quality of life and with inguinal hernia repair, the scores decreased drastically in each of the 8 categories and in total score, which correlates with our hypothesis. The p-values were less than 0.001 in each category, which is statistically significant. Knox et al. (14) had a similar result in their study about quality of life after surgery. Their study confirmed that as there's a crucial change in the quality of life after hernia repair, the postoperative scores were significantly less than the preoperative scores (14). Lawrence et al. (15) also conducted a similar study about quality of life after hernia repair with another quality of life scale called Short Form 36. This form is a global measure of health-related quality of life that measures the physical and mental health perception before and after the operation (16). Their results also demonstrate the improvement in quality of life of patients who had undergone inguinal hernia repair. These values are also statistically significant again correlating with our results.

Only 86 out of 190 patients responded to questions, excluding the rest from the study. However, this exclusion did not seem to affect the results of the study due to the high significance of scores. The main limitation was that all of the patients responded to the questionnaires in postoperative periods. However, a cross-sectional analysis may give a broader and more realistic score, especially for preoperative discomfort.

In conclusion, our study showed that inguinal hernia repair with mesh makes a crucial improvement in patients' quality of life regarding different daily activities.

Ethics Committee Approval: This retrospective study was approved by the Scientific Research Ethics Committee of Trakya University School of Medicine (TUTF-BAEK2019/384.

Informed Consent: Written informed consent was obtained from the participants of this study.
Conflict of Interest: The authors declared no conflict of interest. Author contributions: Concept: EC, TKA, HSÇ, TD. Supervision: EC, TKA, HSÇ, TD. Resources: EC, TKA, HSÇ, TD. Materials: EC, TKA, HSÇ, TD. Data collection and/or processing: EC, TKA, HSÇ, TD. Analysis and/or Interpretation: EC, TKA, HSÇ, TD. Literature Search: EC, TKA, HSÇ, TD. Writing Manuscript: EC, TKA, HSÇ, TD. Critical Review: EC, TKA, HSÇ, TD.

Financial disclosure: The authors declared that this study received no financial support.

Editor-in-chief's Note: Two authors of this article, Elif Cengiz, Hilal Sena Çifcibaşı are members of the editorial board of Turkish Medical Student Journal. However, they did not take place in any stage on the editorial decision of the manuscript. The editors who evaluated this manuscript are from other institutions.

\section{REFERENCES}

1. Jenkins JT, O'Dwyer PJ. Inguinal hernias. BMJ 2008;336(7638):26972.

2. Hammoud M, Gerken J. Inguinal hernia. StatPearls (serial online) 2019 Jan (cited 2019 Feb). Available from: URL: https://www.ncbi. nlm.nih.gov/books/NBK513332/?report=reader\#_NBK513332_ pubdet_.

3. HerniaSurge Group. International guidelines for groin hernia management. Hernia 2018;22(1):1-165.

4. Sharma P, Boyers D, Scott $\mathrm{N}$ et al. The clinical effectiveness and cost-effectiveness of open mesh repairs in adults presenting with a clinically diagnosed primary unilateral inguinal hernia who are operated in an elective setting: systematic review and economic evaluation. Health Technol Assess 2015;19(92):1-142.

5. Naguib N, ElSamerraai A. No-mesh inguinal hernia repair with continuous absorbable sutures: is it a step forward or backward?. Saudi J Gastroenterol 2009;15(1):68-9.

6. Bringman S, Ramel S, Heikkinen TJ et al. Tension-free inguinal hernia repair: TEP versus mesh-plug versus Lichtenstein: a prospective randomized controlled trial. Annals of surgery 2003;237(1):1427.

7. Rutegård M, Gümüsçü R, Stylianidis G et al. Chronic pain, discomfort, quality of life and impact on sex life after open inguinal hernia mesh repair: an expertise-based randomized clinical trial comparing lightweight and heavyweight mesh. Hernia 2018;22(3):411-18.

8. Sanders DL, Waydia S. A systematic review of randomised controled trials assessing mesh fixation in open inguinal hernia repair. Hernia 2014;18:165-176.

9. Heniford BT, Walters AL, Lincourt AE et al. Comparison of generic versus specific quality-of-life scales for mesh hernia repairs. J Am Coll Surg 2008;206(4):638-44.

10. Turaga K, Fitzgibbons RJ, Puri V. Inguinal hernias: should we repair? Surg Clin North Am 2008;88:127-38.

11. Primatesta P, Goldacre MJ. Inguinal hernia repair: incidence of elective and emergency surgery, readmission and mortality. Int J Epidemiol 1996;25:835-9. 
12. Burcharth J, Pedersen M, Bisgaard T et al. Nationwide prevalence of groin hernia repair. PLoS One 2013;8(1): e54367.

13. Öberg S, Andresen K, Rosenberg J. Etiology of inguinal hernias: acomprehensive review. Front Surg 2017;4(52):1-8.

14. Knox RD, Berney CR. A preoperative hernia symptom score predicts inguinal hernia anatomy and outcomes after TEP repair. Surg Endosc 2015;29(2):481-6.

15. Lawrence K, McWhinnie D. Quality of life in patients undergoing inguinal hernia repair. Ann R Coll Surg Engl 1997;79(1):40-5.

16. Lins L, Carvalho FM. SF-36 total score as a single measure of health-related quality of life: scoping review. SAGE Open Medicine 2016;4:1-12. 